\title{
EJNSO
}

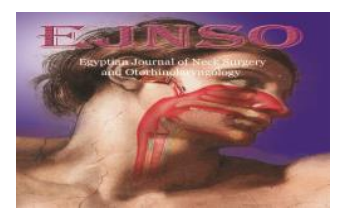

\section{Evaluation of audio-vestibular functions in patients with open angle glaucoma}

Abeer Ali ${ }^{1,2}$, Enas Sayed Mohamed ${ }^{2}$, Amira Mohammad Eloseily ${ }^{2}$

1.Unit of Audio-vestibular Medicine, South Valley University, Qena, Egypt

2.Unit of Audio-vestibular Medicine, Assiut University, Assiut, Egypt.

\section{Abstract:}

Introduction: Visual and hearing impairment compromise the basic modes of communication leading to social isolation and impaired cognitive and general functions. Inconsistent results regarding the co-existence of eye and ear pathologies have been found .

Objectives: The aim of this study is to assess audio-vestibular functions in patients with open angle glaucoma (OAG) .

Patients and Methods: Fifty patients with OAG and 20 age and sex-matched healthy controls were included. Pure tone audiometry, immitencemetry, distortion product otoacoustic emission (DPOAEs), auditory brainstem response (ABR), and electronystagmography (ENG) were done and compared between the two groups.

Results: Compared to the control group, patients with OAG had significantly elevated pure tone audiometric thresholds at all audiometric frequencies ( $\mathrm{P}$ between 0.003 to 0.001 ), sensory neural hearing loss (SNHL) in $86 \%$ of the patients, lower amplitude of the DPOAEs at all tested frequencies, prolonged waves I, III and V in ABR and abnormal caloric test in $22 \%$ of the patients.

Conclusions: The present study confirmed the association between SNHL and OAG. The results of PTA, DPOAEs and caloric tests suggesting that the cochlea is most probably the main site of pathology in patients with OAG.

Key words: Open angle glaucoma, Sensory neural hearing loss.

\section{Introduction}

Sensory neural hearing loss (SNHL) refers to hearing impairment due to damage of the cochlea, auditory nerve or central nervous system. Age-related SNHL represents more than $60 \%$ in those older than 65 years. Other causes of SNHL include noise-related, infectious, autoimmune, inner ear diseases, ototoxic drugs or vascular causes. ${ }^{1}$ Additionally, acoustic neuroma and central nervous system lesions like multiple sclerosis are wellknown causes of SNHL. ${ }^{2}$
Glaucoma is an optic neuropathy characterized by changes to the optic nerve head morphology, accompanied by thinning and specific loss of retinal ganglion cells (RGCs). It is a leading cause of irreversible vision loss worldwide. Primary open-angle glaucoma (POAG), the most common form of the disease, accounts for the majority $(74 \%)$ of glaucoma cases. In 2013, more than 44 million individuals were affected by POAG. ${ }^{3}$ Age is a strongly established risk factor for 
POAG, with prevalence in US adults increasing from $0.6 \%$ at ages $40-49$ to $8.3 \%$ at age 80 or older. ${ }^{4}$

The exact pathophysiology of glaucomatous optic neuropathy is incompletely understood; however, there is accumulating evidence for multiple causes, such as mechanical stress and alterations in ocular blood flow, that impinge upon RGC survival with some RGCs being more susceptible to injury than others. ${ }^{5}$

A lot of the researchers investigated nonvisual sensory deficits in the relationship between the eye and ear pathologies. It was discovered back in $1889^{6}$ when a general disease affects the eye, ear involvement is also likely, and vice versa. As far as the coexistence of eye and ear pathologies is concerned, different clinical researchers have come to different conclusions. Seth and Dayal ${ }^{6}$, who evaluated the audiovestibular functions in glaucoma patients, found a cochlear lesion in all of their patients with established primary glaucoma within two years of glaucoma diagnosis. Vick and Vick ${ }^{7}$ and Berkowitz et al ${ }^{8}$, on the other hand, could not establish a definite coincidence of the two lesions in similar investigations. Open angle glaucoma (OAG) is a neurodegenerative condition. Individuals who are susceptible to autonomic neural injury are at high risk for developing it. Data support the plausibility of auditory neural impairment in a proportion of individuals with $\mathrm{OAG}$, with results showing a major disruption to processing temporal properties of the sound. ${ }^{9}$

Previous studies that investigated sensory deficits in OAG has focused on the incidence of hearing loss in association with glaucoma, with findings being inconsistent and disputed across OAG subtypes, namely, the variable incidence in high- and low-tension groups. ${ }^{10}$ The theory of global neuronal vulnerability in OAG predicts abnormalities in the function of the auditory neural pathway despite of the presence of adequate cochlear hair cell function. ${ }^{11}$ However, there is an evidence supports that sensorineural hearing loss resulting from a deficit at the cochlear level is present in a secondary cause of OAG, specifically in patients with Pseudoexfoliation, where the hearing loss is due to the accumulation of extracellular matrix within the inner ear. $^{12}$

The aim of the present study is to evaluate the audio-vestibular functions in patients with open angle glaucoma.

\section{Patients and Methods:}

This was a prospective crosssectional study. The study protocol was reviewed by the Office of Research Enterprise, the institutional review board (IRB) of the Assiut University. The research was conducted in accordance with the tenets of the Declaration of Helsinki. All included participants provided informed consent for inclusion in the study.

\section{Patients:}

Fifty patients with OAG and 20 healthy controls were included in this study. Patients were recruited from the ophthalmic clinic at Assiut University Hospital. The healthy controls were recruited from the relatives accompanying patients in the AudioVestibular unit at Assiut University Hospital.

Inclusion criteria for healthy controls were:

1) Normal ocular examination.

2) Bilateral within normal hearing sensitivity 
The inclusion criteria for glaucoma patients were

1) Diagnosis with OAG based on an ocular examination including anterior eye examination, gonioscopy, measurement of intraocular pressure, optic nerve head examination and automated perimeter.

The exclusion criteria were

1) The presence of other ocular diseases or neurologic disorders that cause similar visual field defects.

2) Previous or current middle ear disease, presence of conductive hearing loss, presence of known causes of SNHL such as systemic disorders like diabetes and hypertension, history of ototoxic drugs intake and presence of known inner ear disorder such as Meniere disease, autoimmune inner ear disease or chronic noise exposure.

\section{Ophthalmological evaluation:}

All participants underwent a complete clinical evaluation, including visual acuity, refraction, anterior segment examination using slit lamp, Goldmann applanation tonometry to determine intraocular pressure (IOP), gonioscopy to determine whether angles are open or closed, fundus examination for optic nerve head evaluation, perimetry with Humphrey perimeter using full threshold algorithm to establish the glaucomatous damage.

These patients were diagnosed as OAG when the IOP was high with open angles on gonioscopy and glaucomatous changes on clinical optic nerve evaluation. The changes in the nerve were substantiated with corresponding perimetric changes.

\section{Audiovestibular evaluation:}

All participants in the current work were subjected to the following:

\section{1- Full history taking:}

History included hearing loss, tinnitus, vertigo, presence of nausea, vomiting and a description of the vertiginous attack, frequency of attack, duration of each attack, condition of the patients in between attacks.

\section{2- Otoscopic examination.}

3- Immittancemetry using Interacoustic immittance meter (AT235 Denmark) to evaluate middle ear function.

4- Pure tone and speech audiometry using audiometer Madsen Orbiter 922 and sound-treated room (amplisilence) to assess hearing sensitivity. Air conduction threshold was obtained for the frequency range $250-8000 \mathrm{~Hz}$ at single octave intervals using a TDH 49 earphone, while bone conduction threshold was obtained for the frequency range $500-4000 \mathrm{~Hz}$ at single octave intervals using a B71 bone vibrator. Speech reception threshold (SRT) and speech discrimination scores were measured using the bisyllabic and monosyllabic phonetically balanced word, respectively. ${ }^{13}$

5- Distortion Product Otoacoustic Emission was recorded using the Intelligent Hearing system (IHS); two channels evoked potential recording apparatus with Smart OAE 4.5 software. Two tones were used: L1 $=65 \mathrm{~dB}$ SPL and L2 $=55 \mathrm{~dB}$ SPL, while f2/f1 was 1.22 . Both the amplitude of response of the distortion product (D.P.) at $2 \mathrm{f} 1-\mathrm{f} 2$ and background noise (Ns) were obtained at nine points corresponding to $\mathrm{f} 2$ frequencies of 553, 783, 1105, 1560, 2211, 3125, 4416, 6250 and $8837 \mathrm{~Hz}$. These measurements were used to build a DP-gram by displaying the D.P. against the $\mathrm{f} 2$ frequency. The signal 
to noise ratio (SNR) was measured (SNR=DP-Ns) at each of these nine points. DPOAE was considered normal (intact), thereby reflecting normal cochlear function, if the SNR was > 3dB SPL on at least $70 \%$ of the tested frequencies. DPOAE was considered abnormal if SNR was > 3dB SPL in less than $70 \%$ of the tested frequencies or if SNR $<3 \mathrm{~dB}$ SPL in all tested frequency. ${ }^{14}$

6- Auditory brainstem response for neural assessment: using IHS two channels evoked potential apparatus with Smart E.P.s software, version 4.5. This was done through twochannel recording using three disposable electrodes applied to the following sites: high frontal $\mathrm{Fz}$ (positive electrode), ipsilateral mastoid (negative electrode), and common electrode were placed on the contralateral mastoid. All electrodes were connected to the pre-amplifier of the Smart-EP equipment. ABR was recorded ipsilaterally in response to click stimuli presented at $90 \mathrm{~dB} n$ H.L. using alternating polarity at 21.1 and 71.1 repetition rates. Stimuli were delivered via TDH 39 headphones. The response was filtered between 100 and $3000 \mathrm{~Hz}$, amplified 100,000 times, recorded over $10.24 \mathrm{~ms}$ time window, and 2000 sweeps were averaged for each run. The absolute latencies of wave I, III and V, interpeak latencies (IPLs) I-III, III-V and I-V as well as the IAL difference of waves III and V were calculated. Absolute latencies and interpeak latencies were considered abnormal if they were more than mean +2.5 $\mathrm{SD}$ of the control values. ${ }^{15}$ Interaural latency difference was considered abnormal if it was more than 2 ms. ${ }^{16}$
7- Electronystagmography: using micromedical tech, Meta 4 apparatus software version4.5.

The subject sat on the test chair one meter away from the center of the light. Then, alcohol swabbing of the skin of the face at the electrode sites was used before placement of the electrodes.

A pair of electrodes was placed near the subsect's outer canthi for monitoring horizontal eye positions. Vertical eye position was monitored by placing a pair of electrodes above and below one eye in the plain of the pupil.

A fifth electrode was placed in the forehead as aground. Electrode impedance should not exceed 5000 ohms, the room light then was turned off.

Eye signal calibration was done by asking the participant to look to left and right, then looked up and down at a moving target with known angle $\left(20^{\circ}\right.$ visual angle) for horizontal and vertical calibration.

ENG examination included searching for spontaneous nystagmus with fixation and with removal of fixation. It also included searching for gaze-evoked nystagmus with the eyes in a central position, on the horizontal $\left(30-35^{\circ}\right.$ to the right and left) and vertical $\left(25-30^{\circ}\right.$ up and down) planes. The test also included recording the smooth pursuit eye movement and saccadic eye movement.

The search for positioning and positional nystagmus was also performed during the right and left Dix-Hallpike tests, and when patients were in the supine position (with the head centered, head to the right and head to the left). (Ice-cold) caloric testing was performed using water irrigation of $2 \mathrm{ml}$ for 30 seconds at $10^{\circ} \mathrm{C}^{17}$

The slow-phase velocity (SPV) measure was used to calculate the canal weakness. Canal weakness was 
calculated through such formula (larger ear response - smaller ear response / total $\times 100$ ). The response was considered as canal weakness if asymmetry more than $20 \%{ }^{18}$

\section{Statistical analysis:}

Patient demographics were summarized by the mean and standard deviation (S.D.). Normality was assessed for all analyses, and appropriate non-parametric methods were applied as necessary.

The comparisons between study and control groups were assessed using an independent sample student t-test. Statistical analysis was carried out using SPSS software (version 24.0, SPSS Inc., Chicago, USA). A p-value of less than 0.05 was considered statistically significant.

\section{Results:}

$\begin{array}{lr}\text { Patient } & \text { characteristics } \\ \text { associated } & \text { audiovestibular } \\ \text { symptoms: } & \end{array}$

Fifty patients (40 females) with OAG and 20 healthy subjects (16 females) were recruited. The mean age was $55 \pm 8.7$ for glaucoma patients and
$53 \pm 9.5$ for healthy participants. Amongst the 50 OAG patients, $7 \%$ had a history of hearing loss, $38 \%$ had a history of vertiginous attack, $20 \%$ had a sensory imbalance, $44 \%$ had tinnitus, and $14 \%$ had drop attacks, $52 \%$ had a positive family history of OAG.

\section{Pure tone audiometry and speech discrimination:}

There was a statistically significant difference in the mean of hearing sensitivity between the glaucoma group and the control group at all tested frequencies, as shown in Table 1.

In glaucoma group, speech discrimination was excellent in 57 ears (57\%), good in 36 ears (36\%), and fair in 7 ears (7\%) while in control group it was excellent in all ears.

Figure1 summarizes the mean of hearing sensitivity between the glaucoma group and the control group at all tested frequencies.

Figure (1): show the mean of hearing sensitivity in glaucoma and control group at different frequencies

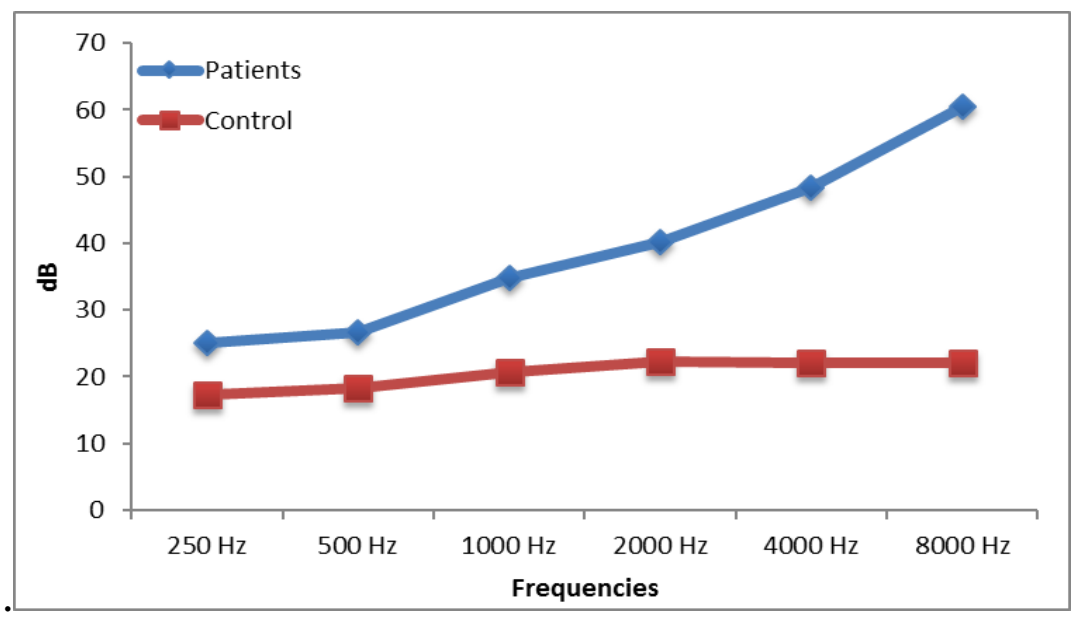


Table 1. Comparison between glaucoma group and control group regarding hearing sensitivity at different frequencies.

\begin{tabular}{|c|c|c|c|c|c|c|}
\hline \multirow[b]{2}{*}{$\begin{array}{l}\text { Frequencies } \\
\qquad(\mathrm{Hz})\end{array}$} & \multicolumn{3}{|c|}{ Right ear } & \multicolumn{3}{|c|}{ Left ear } \\
\hline & $\begin{array}{r}\text { Glaucoma } \\
\text { group }\end{array}$ & $\begin{array}{r}\text { Control } \\
\text { group }\end{array}$ & $P$ value & $\begin{array}{r}\text { Glaucoma } \\
\text { group }\end{array}$ & $\begin{array}{c}\text { Control } \\
\text { group }\end{array}$ & $P$ value \\
\hline 25 & 25 & $183(47$ & 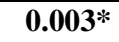 & 24 & 16. & te \\
\hline 5 & 27. & 18. & $<0.0$ & .2) & 18 & $<0.001 *$ \\
\hline 1000 & $33.7(10.7)$ & 21.3 & $<0.001 *$ & 35.9 & $20(3.6)$ & $<0.001 *$ \\
\hline 2000 & 38.2 & 22 & $<0.001 *$ & 42.1 & $22.3(3.4)$ & $<0.001 *$ \\
\hline 4000 & $47.7(15.4)$ & 22 & $<0.001 *$ & 49.1 & $22(3)$ & $<0.001 *$ \\
\hline 8000 & $58.7(18.4)$ & $22(3.4)$ & $<0.001 *$ & $62.3(21.9)$ & $22.3(3)$ & $<0.001 *$ \\
\hline
\end{tabular}

Data is expressed in mean and (SD).

* Statistically significant

\section{Distortion product otoacoustic} emissions (DPOAEs):

There was a statistically significant difference in DPOAEs amplitude and SNR between patients with glaucoma and healthy controls at different tested frequencies as shown in Table $\mathbf{2 \& 3}$ respectively.

\section{Auditory brainstem response(ABR) :}

Abnormal ABR response was found in $64 \%$ of patients ears with 14 ears (14\%) had prolonged wave I, eight ears (8\%) had prolonged wave III, six ears $(6 \%)$ had prolonged wave $\mathrm{V}$, eight ears
(8\%) had prolongation in wave III-V. However, none of them had abnormal prolongation of wave $\mathrm{V}$ at a high repetition rate (R.R.). Table 4 illustrates the interaural latency of wave I, wave III, and wave V in glaucoma and control group.

\section{Electronystagmography:}

Normal electrophysiological test results were found in all participants except for the caloric response, which showed unilateral weakness in $22 \%$ of ears of glaucoma patients.

Table 2. Comparison between the glaucoma group and the control group regarding DPOAEs amplitude at different frequencies

\begin{tabular}{|c|c|c|c|c|c|c|}
\hline \multirow[b]{2}{*}{$\begin{array}{c}\text { Frequencies } \\
(\mathrm{Hz})\end{array}$} & \multicolumn{3}{|c|}{ Right ear } & \multicolumn{3}{|c|}{ Left ear } \\
\hline & $\begin{array}{r}\text { Glaucoma } \\
\text { group }\end{array}$ & $\begin{array}{r}\text { Control } \\
\text { group }\end{array}$ & $P$ value & $\begin{array}{c}\text { Glaucoma } \\
\text { group }\end{array}$ & $\begin{array}{c}\text { Control } \\
\text { group }\end{array}$ & $P$ value \\
\hline 533 & $5.5(8.1)$ & $3.8(1,5)$ & $0.042 *$ & $4.7(9)$ & $1.2(0.2)$ & $0.004 *$ \\
\hline 783 & $3.8(2.4)$ & $5.5(3.2)$ & 0.031* & $4.8(6)$ & 6.1(4.6) & $0.004 *$ \\
\hline 1105 & $1.2(6.8)$ & $9.7(4)$ & $<0.001^{*}$ & $2.7(1.7)$ & $8.3(3.6)$ & $<0.001 *$ \\
\hline 1560 & $9.5(2.5)$ & $8.7(4.8)$ & $<0.001 *$ & $8.1(2)$ & $7.2(3.8)$ & $0.021 *$ \\
\hline 2211 & $4.3(1.5)$ & $8.4(3.3)$ & $<0.001 *$ & $2.9(4.5)$ & $6.9(1.4)$ & $0.010 *$ \\
\hline 3125 & $6.3(2.1)$ & $4.8(4)$ & $<0.001 *$ & $9.8(7.5)$ & $5.5(2.5)$ & $0.003 *$ \\
\hline 4416 & $6.6(3.2)$ & $6.1(4.9)$ & $<0.001 *$ & $8.5(6.8)$ & $3.7(4.7)$ & $0.036 *$ \\
\hline 6250 & $5.2(6.4)$ & $4(2.2)$ & $0.041 *$ & $9.2(8.9)$ & $1.7(4.4)$ & $<0.001 *$ \\
\hline 8837 & $9.2(7.8)$ & $6.2(1.1)$ & $0.028 *$ & $8.6(6.4)$ & $6.6(3.8)$ & $0.010 *$ \\
\hline
\end{tabular}

Data is expressed in mean and (SD).

* Statistically significant 
Table 3. Comparison between the glaucoma group and control group regarding DPOAEs SNR at different frequencies

\begin{tabular}{|c|c|c|c|c|c|c|}
\hline \multirow[b]{2}{*}{$\begin{array}{l}\text { Frequencies } \\
\qquad(\mathrm{Hz})\end{array}$} & \multicolumn{3}{|c|}{ Right ear } & \multicolumn{3}{|c|}{ Left ear } \\
\hline & $\begin{array}{r}\text { Glaucoma } \\
\text { group }\end{array}$ & $\begin{array}{r}\text { Control } \\
\text { group }\end{array}$ & $P$ value & $\begin{array}{r}\text { Glaucoma } \\
\text { group }\end{array}$ & $\begin{array}{r}\text { Control } \\
\text { group }\end{array}$ & $P$ value \\
\hline 533 & $6.1(3.5)$ & $7.3(2.9)$ & 0.028* & $6.2(8.4)$ & $9.7(7.1)$ & 0.004* \\
\hline 783 & $5.2(3.2)$ & $11.5(4.1)$ & $<0.001 *$ & $6.1(6.4)$ & $7.4(6.1)$ & 0.004* \\
\hline 1105 & $8.3(4.1)$ & $12.2(5.1)$ & $<0.001 *$ & $8.4(6)$ & 11.7(7.9) & $<0.001 *$ \\
\hline 1560 & $9.1(6.1)$ & $17.7(6.7)$ & $<0.001 *$ & $8.8(10.9)$ & $10.2(9.5)$ & 0.021* \\
\hline 2211 & $9.3(6.3)$ & $13.4(4.5)$ & 0.013* & $6.9(9.9)$ & $10.6(1.4)$ & 0.010* \\
\hline 3125 & $5.9(7.8)$ & $12.4(5.2)$ & $<0.001 *$ & $6.2(7.7)$ & $8.2(6.1)$ & 0.003* \\
\hline 4416 & $6.4(5.5)$ & $10.2(9.9)$ & $0.012 *$ & $7.1(5.2)$ & $8.4(6.2)$ & $0.036 *$ \\
\hline 6250 & $5.2(4.5)$ & $8(5.2)$ & $0.011 *$ & $4.5(6.3)$ & $7.7(8.5)$ & $<0.001 *$ \\
\hline 8837 & $3.1(5.9)$ & $10.2(6.8)$ & 0.012* & $2.6(8.4)$ & 8.2(1.1) & 0.010* \\
\hline
\end{tabular}

Data is expressed in mean and (SD)

*.Statistically significant

Table 4. Comparison between glaucoma group and control group interaural latency of waves I, III, and V.

\begin{tabular}{llll}
\hline IAL & $\begin{array}{l}\text { Glaucoma } \\
\text { group }\end{array}$ & $\begin{array}{l}\text { Control } \\
\text { group }\end{array}$ & P-value \\
\hline IAL I & $\mathbf{0 . 1 6}(0.1)$ & $\mathbf{0 . 0 9}(0.1)$ & $\mathbf{0 . 0 1}^{*}$ \\
IAL III & $\mathbf{0 . 1 5}(0.2)$ & $0.07(0.1)$ & $\mathbf{0 . 0 3}^{*}$ \\
IAL V & $0.17(0.1)$ & $0.11(0.1)$ & $\mathbf{0 . 0 3}^{*}$ \\
\hline
\end{tabular}

Data is expressed in mean and (SD).

* Statistically significant

\section{Discussion:}

Visual impairment impacts both physical and social functions, whereas hearing impairment mainly affects social function. ${ }^{19}$ Dual sensory impairment, hearing loss and visual loss, compromise the basic modes of communication, listening, speech and reading, potentially leading to social isolation and impaired cognitive and general functions. ${ }^{20}$

Although there is no known clear sex predilection for $\mathrm{OAG},{ }^{21}$ several population-based prevalence studies conducted in different regions of the world have reported inconsistent results regarding the prevalence of POAG between genders. The Baltimore Eye Survey 22, the Melbourne Visual Impairment Project ${ }^{23}$, and the Projecto VER ${ }^{24}$ showed no difference in prevalence by gender. In contrast, the Blue Mountain Eye Study revealed that the age-adjusted prevalence of glaucoma was higher in women, although this finding was of borderline significance. ${ }^{25}$ However, the Barbados Eye Study ${ }^{26}$, the Framingham Eye Study $^{27}$, the Rotterdam Study ${ }^{28}$, and the Los Angeles Latino Eye Study found a higher prevalence of glaucoma among men compared with women ${ }^{29}$. The current study revealed female predominance in the OAG (Female to male ratio was $4: 1)$. These conflicting results may be due to varying definitions of glaucoma, selection of study sample, reporting bias, and other contributing factors such as women live longer than men, so they have increased risk of developing glaucoma. 30 The present study showed that $52 \%$ of patients with OAG have a positive family history. This is consistent with the fact that around half of all primary OAG patients have a positive family history. ${ }^{31}$

Current study showed a significant association between SNHL and OAG. 
Patients with OAG had significantly elevated pure tone audiometric thresholds when compared to control subjects at all audiometric frequencies. SNHL was present in $86 \%$ of OAG patients, in those patients, SNHL was bilateral either of mild, moderate, or severe degree.

In agreement with the other clinical studies that revealed a common presence of SNHL in OAG patients. Seth and Dayal ${ }^{6}$ used conventional audiometric tests to investigate glaucoma patients. Although only 23\% of their glaucoma patients had manifested signs of Mèniére's disease, inner ear involvement was found in all patients with glaucoma of more than two years duration. In patients with primary glaucoma, hearing impairment was usually bilateral.

Cahill et al ${ }^{32}$ examined hearing thresholds 1, 2, $3 \mathrm{kHz}$ and concluded that most pseudoexfoliation syndrome (XFS)/Pseudoexfoliation glaucoma (XFG) individuals seemed to have a worse hearing when compared to the age- and sex-matched controls.

Yazdani et al ${ }^{33}$ suggested that hearing thresholds of XFS/XFG cases maybe around $10 \mathrm{~dB}$ H.L. worse for both lower $(1 \mathrm{kHz})$ and middle/higher frequencies ( 2 and $3 \mathrm{kHz}$ ) compared to Papadopoulos et al ${ }^{34}$ who found, contrary to the above-mentioned studies, the strongest association at the highest frequency, $8 \mathrm{kHz}$, and no association with thresholds at frequencies of $0.25,0.5,1$, and $2 \mathrm{kHz}$. Paliobei et al ${ }^{35}$ published on hearing and exfoliation syndrome, including 110 patients with XFG as well as 85 patients with POAG, mean age 66 years, range 50-70 years, examining auditory thresholds of $0.5,1,2,4$ and 8 $\mathrm{kHz}$. They showed poorer hearing in the XFG group for all frequencies compared with the controls.

In the current work, DPOAEs were abnormal in $86 \%$ of the ears of OAG patients. these results were consistent with pure tone audiometric results suggesting that cochlear affection is common among OAG patients. Previous studies reported the association between cochlear affection and some ocular conditions. ${ }^{35-37}$

Cagini et al ${ }^{37}$ found reduced DPOAE amplitudes in retinitis pigmentosa patients with normal hearing. Heaton and Mills ${ }^{38}$ found that there was fluctuating hearing loss in patients with Birdshot chorioretinopathy, which probably due to endolymphatic hydrops (E.H.). The authors suspected a possible relationship between the sensory cells in Corti's organ and the retinal photoreceptors. Vargas 39 and Moorthy et al ${ }^{40}$ suggested that Vogt Koyanagi-Harada syndrome abnormalities (uveitis, vitiligo, and dysacusis) may result from an autoimmune mechanism directed against the cochlear and visual pigment derived from the neural crest; glaucoma was found in $33 \%$ of these patients that demonstrated abnormal DPOAEs.

In the current study, there was a statistically significant prolongation of the ABR waves I, III, and V in OAG patients when compared to the control subjects. These results are consistent with another study that showed similar findings. ${ }^{35}$ Results indicate an abnormal delay in the transmission of sound within the auditory brainstem and reflect auditory brainstem dysfunction in $\mathrm{OAG}$ patients. In the current study, the ABR abnormality in the wave I was much more common than the abnormality in the central waves (waves III and V). This is consistent with the predominance of cochlear pathology in OAG patients. Although the mean latency of wave III, and $\mathrm{V}$ were statistically significant longer in OAG patients when compared to the control subjects, this 
prolongation in the waves did not reach the clinical abnormality (2.5 \pm S.D. above the mean of the control subjects) except only in the minority of ears (2$6 \%$ ).

In the current study, there was a statistically significant prolongation of the ABR waves I, III, and $\mathrm{V}$ in $\mathrm{OAG}$ patients when compared to the control subjects. These results are consistent with another study that showed similar findings. ${ }^{35}$ Results indicate an abnormal delay in the transmission of sound within the auditory brainstem and reflect auditory brainstem dysfunction in OAG patients. In addition, there was no significant difference between the study group and the control group as regard the ABR inter-peak latencies. Similarly, there was no statistically significant difference between the study group and the control group as regard the interaural latency difference of wave III and $\mathrm{V}$ reflecting symmetrical ABR abnormality in the OAG patients.

Previous studies ${ }^{41-42}$ demonstrated the presence of unilateral caloric weakness in patients with OAG. They reported an evidence of similar structural and biochemical mechanisms that lead to malproduction and malresorption of aqueous humor in the ciliary body and trabecular meshwork and endolymph in the cochlea, suggesting the association of glaucoma and E.H. In the current study, the only ENG abnormality found was the caloric test which was unilaterally weak in $(22 \%)$ of ears. The small sample size limited proper statistical analysis, however, observation of data suggested an association between OAG patients and caloric weakness as compared to the control group. A possible explanation of unilaterality of the caloric weakness in this work could be related to the fact that OAG is a bilateral asymmetrical disease. Therefore, one ear might be affected more than the other, possibly due to the presence of endolymphatic hydrops. According to Bhansali et al ${ }^{43}$, the most frequent abnormality noticed in patients with endolymphatic hydrops is a unilateral weakness in caloric testing. In addition, Paparella et al ${ }^{44}$ reported that up to $50 \%$ of patients with Meniere disease have normal caloric test even in the presence of incapacitating vestibular symptoms.

The connection between hearing loss and glaucoma is interesting, because the cells in the ear that produce and drain endolymph are structurally analogous to the anterior segment cells in the eye that produce and drain aqueous humor. 43 Ultrastructural findings showed that fibrils similar to those seen in XFG have been found in the basement membranes. ${ }^{44}$

Several reports have suggested that the accumulation of exfoliation material aggregates in the vessel walls is associated with an increased risk for vascular disease. There is a common embryological origin between the anterior segments of the eye and the tectorial membrane of the inner ear from neural ectoderm, and their structure similarly includes type $\mathrm{V}$ collagen fibers. If the organ of Corti is also involved in XFG and is a site for exfoliation deposition, this could disrupt its chemical environment and result in sensorineural hearing loss. ${ }^{36}$ This process conceivably may lead to a change of fluid homeostasis, which could cause tinnitus or even endolymphatic hydrops. ${ }^{44}$ The latter hypothesis was supported by the findings of Turgut et al. ${ }^{12}$.

Several studies have proved enzyme activities that affect both ear and eye. Carbonic anhydrase is an enzyme that has been reported to exist in the kidney, eye, inner ear, and some other tissues. It is distributed throughout the ciliary body in the pigmented and nonpigmented epithelium ${ }^{45}$ and in the 
retinal pigment epithelium of the eye.

46 Carbonic anhydrase also plays an important role in the regulation of inner ear fluid dynamics and can be found in most cells of the cochlear duct. ${ }^{47}$

It has been found that in guinea pigs with E.H., produced by obliteration of the endolymphatic duct and sac, acetazolamide leads to a reduction of E.H. during the treatment. ${ }^{42}$. Acetazolamide is applied for treatment E.H. ${ }^{48}$ and glaucoma ${ }^{41}$. Since both diseases have common risk factors, neurodegenerative features, and comorbidities, it is accepted that patients with SNHL have a sensitive nervous system in which the optic nerve could be affected more easily than the normal population.

\section{Conclusions:}

In conclusion the present study confirmed the association between SNHL and OAG. The results of PTA, DPOAEs and caloric tests suggesting that the cochlea is most probably the main site of pathology in patients with OAG.

\section{Recommendation:}

We recommend adding VEMP recording to the test battery of OAG patients for evaluation of the integrity of the sacculo-colic reflex.

Transient evoked autoacoustic emission (TEOAE) could be also done in combination with DPOAE for more accurate assessment of cochlear function.

We also recommend applying auditory tests in the clinical evaluation of patients with OAG, so patients with abnormal results can be treated as having dual sensory loss with specific rehabilitation management.

\section{Limitations:}

There are few limitations to our study, the overall number of patients included in this study was small and we did not compare different types of glaucoma with normal controls and among each other so further larger studies are recommended.

\section{Financial support and sponsorship:}

No financial support was obtained from any source.

\section{Conflicts of interest:}

The authors declared no potential conflicts of interest with respect to the research, authorship and/or publication of this article.

\section{Reference:}

1. Chien, H.W., et al., Increased Incidence of Glaucoma in Sensorineural Hearing Loss: A Population-Based Cohort Study. Int J Environ Res Public Health, 2019. 16(16).

2. Di Stadio, A., et al., Sudden hearing loss as an early detector of multiple sclerosis: a systematic review. Eur Rev Med Pharmacol Sci, 2018. 22(14): p. 4611-4624.

3. Quigley, H.A. and A.T. Broman, The number of people with glaucoma worldwide in 2010 and 2020. Br J Ophthalmol, 2006. 90(3): p. 262-7.

4. Gupta, P., et al., Prevalence of Glaucoma in the United States: The 2005-2008 National Health and Nutrition Examination Survey. Invest Ophthalmol Vis Sci, 2016. 57(6): p. 2905-2913.

5. Vrabec, J.P. and L.A. Levin, The neurobiology of cell death in glaucoma. Eye, 2007. 21(1): p. 6702880.

6. Seth, R.S. and D. Dayal, Inner-ear involvement in primary glaucoma. 
Ear Nose Throat J, 1978. 57(8): p. 355-9.

7. Vick, H.P. and U. Vick, [On the problem of the occurrence of glaucoma and cochlear vestibular disorders at the same time]. Acta Ophthalmol, 1970. 48(3): p. 349-56.

8. Berkowitz, W.P., D.G. Sessions, and M.H. Stroud, Letter: Endolymphatic hydrops and glaucoma production. Ann Otol Rhinol Laryngol, 1974. 83(4): p. 5556.

9. O'Hare, F., et al., Auditory and visual temporal processing disruption in open angle glaucoma. Invest Ophthalmol Vis Sci, 2012. 53(10): p. 6512-8.

10. Kremmer, S., et al., Coincidence of normal tension glaucoma, progressive sensorineural hearing loss, and elevated antiphosphatidylserine antibodies. $\mathrm{Br}$ J Ophthalmol, 2004. 88(10): p. 125962.

11. Rance, G., Auditory neuropathy/dys-synchrony and its perceptual consequences. Trends Amplif, 2005. 9(1): p. 1-43.

12. Turgut, B., et al., The evaluation of vestibular functions in patients with pseudoexfoliation syndrome. Eur Arch Otorhinolaryngol, 2010. 267(4): p. 523-7.

13. S, S., Speech discrimination audiometry using Arabic phonetically balanced words. Ain Shams Medical Journal 1967. 27: p. 27-30.

14. Abdala, C. and L. Visser-Dumont, Distortion Product Otoacoustic Emissions: A Tool for Hearing Assessment and Scientific Study. Volta Rev, 2001. 103(4): p. 281-302.

15. Cashman, M.Z. and R.N. Rossman, Diagnostic features of the auditory brainstem response in identifying cerebellopontine angle tumours. Scand Audiol, 1983. 12(1): p. 35-41.
16. Kochanek, K.M., et al., Comparison of 3 ABR Methods for Diagnosis of Retrocochlear Hearing Impairment. Med Sci Monit, 2015. 21: p. 3814-24.

17. A. Sameh Farid, S.M., El-Abd, Maha H, Abou-Elew. , Monothermal caloric test its value in assessment of vestibular function. International Congress Series, 2003. 1240:131924.

18. Houston, H.G. and D.R. Watson, A review of computerized electronystagmography technology. Br J Audiol, 1994. 28(1): p. 41-6.

19. Wallhagen, M.I., et al., Comparative impact of hearing and vision impairment on subsequent functioning. J Am Geriatr Soc, 2001. 49(8): p. 1086-92.

20. Fischer, M.E., et al., Multiple sensory impairment and quality of life. Ophthalmic Epidemiol, 2009. 16(6): p. 346-53.

21. Vajaranant, T.S., et al., Gender and glaucoma: what we know and what we need to know. Curr Opin Ophthalmol, 2010. 21(2): p. 91-9.

22. Tielsch, J.M., et al., Racial variations in the prevalence of primary open-angle glaucoma. The Baltimore Eye Survey. JAMA, 1991. 266(3): p. 369-74.

23. Yamamoto, M. and G. Watanabe, Effects of maternal ageing on developmental defects in mice. Nihon Eiseigaku Zasshi, 1975. 29(6): p. 558-62.

24. Quigley, H.A., et al., The prevalence of glaucoma in a population-based study of Hispanic subjects: Proyecto VER. Arch Ophthalmol, 2001. 119(12): p. 181926.

25. Mitchell, P., et al., Prevalence of open-angle glaucoma in Australia. The Blue Mountains Eye Study. Ophthalmology, 1996. 103(10): p. 1661-9. 
26. Leske, M.C., et al., The Barbados Eye Study. Prevalence of open angle glaucoma. Arch Ophthalmol, 1994. 112(6): p. 821-9.

27. Kahn, H.A., et al., The Framingham Eye Study. II. Association of ophthalmic pathology with single variables previously measured in the Framingham Heart Study. Am J Epidemiol, 1977. 106(1): p. 33-41.

28. Dielemans, I., et al., The prevalence of primary open-angle glaucoma in a population-based study in The Netherlands. The Rotterdam Study. Ophthalmology, 1994. 101(11): p. 1851-5.

29. Doshi, V., et al., Sociodemographic, family history, and lifestyle risk factors for openangle glaucoma and ocular hypertension. The Los Angeles Latino Eye Study. Ophthalmology, 2008. 115(4): p. 639-647 e2.

30. Rudnicka, A.R., et al., Variations in primary open-angle glaucoma prevalence by age, gender, and race: a Bayesian meta-analysis. Invest Ophthalmol Vis Sci, 2006. 47(10): p. 4254-61.

31. Awadalla, M.S., et al., Copy number variations of TBK1 in Australian patients with primary open-angle glaucoma. Am J Ophthalmol, 2015. 159(1): p. 124-30 e1.

32. Cahill, M., et al., Pseudoexfoliation and sensorineural hearing loss. Eye, 2002. 16(3): p. 261-6.

33. Singham, N.V., et al., Association between Ocular Pseudoexfoliation and Sensorineural Hearing Loss. J Ophthalmol, 2014. 2014: p. 825936.

34. Tryggvason, G., et al., Hearing in older adults with exfoliation syndrome/exfoliation glaucoma or primary open-angle glaucoma. Acta Ophthalmol, 2016. 94(2): p. 140-6.
35. Paliobei, V.P., et al., Hearing Evaluation in Patients with Exfoliative and Primary Open-Angle Glaucoma. Otolaryngol Head Neck Surg, 2011. 145(1): p. 125-30.

36. Cahill, M., et al., Pseudoexfoliation and sensorineural hearing loss. Eye (Lond), 2002. 16(3): p. 261-6.

37. Cagini, C., et al., Study of functionality of cochlear outer hair cells in patients with retinitis pigmentosa. Surv Ophthalmol, 1995. 39 Suppl 1: p. S25-32.

38. Heaton, J.M. and R.P. Mills, Sensorineural hearing loss associated with birdshot retinochoroidopathy. Arch Otolaryngol Head Neck Surg, 1993. 119(6): p. 680-1.

39. Vargas, L.M., The black paintings and the Vogt-Koyanagi-Harada syndrome. J Fla Med Assoc, 1995. 82(8): p. 533-4.

40. Moorthy, R.S., H. Inomata, and N.A. Rao, Vogt-Koyanagi-Harada syndrome. Surv Ophthalmol, 1995. 39(4): p. 265-92.

41. Kabudwand, E.A., et al., Lowfrequency masking for detection of endolymphatic hydrops in patients with glaucoma. Hear Res, 1998. 116(1-2): p. 131-6.

42. Shinkawa, H. and R.S. Kimura, Effect of diuretics on endolymphatic hydrops. Acta Otolaryngol, 1986. 101(1-2): p. 43-52.

43. Ritch, R. and U. SchlotzerSchrehardt, Exfoliation syndrome. Surv Ophthalmol, 2001. 45(4): p. 265-315.

44. Streeten, B.W., et al., Pseudoexfoliative fibrillopathy in visceral organs of a patient with pseudoexfoliation syndrome. Arch Ophthalmol, 1992. 110(12): p. 175762.

45. Matsui, H., et al., Membrane carbonic anhydrase (IV) and ciliary epithelium. Carbonic anhydrase activity is present in the basolateral 
membranes of the non-pigmented ciliary epithelium of rabbit eyes. Exp Eye Res, 1996. 62(4): p. 409-17.

46. Wolfensberger, T.J., et al., Membrane-bound carbonic anhydrase in human retinal pigment epithelium. Invest Ophthalmol Vis Sci, 1994. 35(9): p. 3401-7.

47. Hsu, C.J., Ultrastructural study of cytochemical localization of carbonic anhydrase in the inner ear. Acta Otolaryngol, 1991. 111(1): p. 75-84.
48. Ralli, G., et al., [Effect of acetazolamide on Meniere's disease]. Acta Otorhinolaryngol Ital, 1989. 9(5): p. 503-9.

49. Young, Y.H., T.W. Huang, and P.W. Cheng, Assessing the stage of Meniere's disease using vestibular evoked myogenic potentials. Arch Otolaryngol Head Neck Surg, 2003. 129(8): p. 815-8. 